\title{
Frequency and risk factors for recurrent gestational diabetes mellitus in primiparous women: a case control study
}

\author{
Yin-Yu Wang ${ }^{1}$, Ye Liu', Cheng Li ${ }^{1}$, Jing Lin ${ }^{1}$, Xin-Mei Liu ${ }^{1}$, Jian-Zhong Sheng ${ }^{3}$ and He-Feng Huang ${ }^{1,2^{*}}$ (D)
}

\begin{abstract}
Background: To investigate the frequency and risk factors for recurrent gestational diabetes mellitus (GDM) in Chinese primiparous women.

Methods: Case control study. We investigated primiparous women who experienced GDM complications and had a subsequent pregnancy in the same hospital from January, 2012 to January, 2017. Ultimately, 78 women with recurrent GDM and 64 women with no recurrence were included. Clinical characteristics and biochemical parameters such as fasting plasma glucose (FPG), oral glucose tolerance test (OGTT) and lipid profiles were collected from medical records. We used an independent $t$-test and Chi-square test or Fisher's exact test to compare each variable. Univariate and multivariate logistic analyses were used to compute each odds ratio (OR) and 95\% confidence interval (Cl).

Results: The frequency of recurrent GDM was $55 \%$. We found postprandial 1-h glucose at the $75-\mathrm{g}$ OGTT was positively related to GDM recurrence, whereas first-trimester FPG in first pregnancy was negatively related. The first-trimester $\mathrm{HbA} 1 \mathrm{c}$ value was higher in the group with GDM recurrence than in the group with no recurrence, though the difference was not significant. Moreover, the group with GDM recurrence manifested significantly higher first-trimester triglyceride concentrations in subsequent pregnancies; the adjusted ORs $(95 \% \mathrm{Cl})$ were 1.43 (1.09-1.87), 0.24 (0.10-0.63), $3.59(0.93-13.88)$ and 1.89 (1.13-3.16).

Conclusions: GDM recurred in more than half of subsequent pregnancies. Women with lower first-trimester FPG and higher postprandial 1-h glucose in first pregnancy, and with higher first-trimester triglyceride in subsequent pregnancy were at increased risk for GDM recurrence.
\end{abstract}

Keywords: Gestational diabetes mellitus, Recurrence, Risk factor, Biochemical parameter, Chinese primiparous woman

\section{Background}

Gestational diabetes mellitus (GDM) is a common complication in pregnant women and is defined as maternal carbohydrate intolerance first diagnosed during pregnancy [1]. It was reported that the incidence of GDM was $4.3 \%$ in 2009 [2], and it increased to $17.5 \%$ in 2015 in Chinese

\footnotetext{
* Correspondence: huanghefg@sjtu.edu.cn

${ }^{1}$ The International Peace Maternity and Child Health Hospital, Shanghai Jiao Tong University School of Medicine, No. 1961, Huashan Road, Shanghai 200030, China

${ }^{2}$ The Key Laboratory of Reproductive Genetics (Ministry of Education), Women's Hospital School of Medicine Zhejiang University, Hangzhou 310058, People's Republic of China

Full list of author information is available at the end of the article
}

women [3]; the prevalence has kept rising due to a great number of women living a less physically active lifestyle and entering pregnancy as overweight or obese $[4,5]$. GDM is related to short-term and long-term adverse outcomes for both mother and offspring [6], such as pregnancy-induced hypertension, high rate of cesarean delivery, macrosomia, elevated risk of recurrence in subsequent pregnancy [7] and development of diabetes later in life $[8,9]$.

The reported frequency of GDM recurrence ranges widely from 30 to $84 \%$ [10], depending on the different populations studied and the various diagnostic criteria employed [11]. Although two studies reported the

(c) The Author(s). 2019 Open Access This article is distributed under the terms of the Creative Commons Attribution 4.0 International License (http://creativecommons.org/licenses/by/4.0/), which permits unrestricted use, distribution, and 
recurrence rates of GDM in Asia-Pacific countries [12, 13], limited data were correlated to the Chinese population $[11,14]$ because of the implementation of the "one-child policy" in China. Moreover, other studies [15, 16] reported significant differences in GDM incidence among different Asian countries and suggested that it is important to examine Asian subgroups separately. Risk factors related to GDM recurrence also have varied across previous studies. Of note, ethnicity was consistently associated with recurrence of GDM [10, 11]. In addition, advanced maternal age, pregestational obesity, insulin required in the first pregnancy, family history of diabetes in first-degree relatives, weight gain between pregnancies and postprandial glucose value at the oral glucose tolerance test (OGTT) were also strongly correlated with GDM recurrence [17]. However, other biochemical parameters such as triglycerides and fasting plasma glucose (FPG) during early pregnancy have not been widely evaluated as risk factors of GDM recurrence. In this study, we used a population of women delivering two sequential live singleton infants to examine the recurrence of GDM between parity one and parity two pregnancies.

Taken together, we conducted a case-control study to determine the frequency and risk factors associated with GDM recurrence in Chinese primiparous women, and documented first-trimester biochemical parameters. Our present study aimed to identify additional modifiable risk factors and help these high-risk women reduce the side effects that accompany subsequent GDM.

\section{Methods}

\section{Patients}

The subjects in the present study were all women with GDM between January, 2012 to June, 2015 and had a subsequent pregnancy between May, 2014 to January, 2017 at the International Peace Maternity and Child Health Hospital (IPMCH), Shanghai, China. Women with preexisting diabetes before pregnancy were excluded from this analysis. Women were divided into two groups based on the results of the 75-g OGTT in the subsequent pregnancy: the recurrence group and the group with no recurrence.

\section{Diagnostic criteria}

The diagnostic criteria of GDM were made according to the International Association of the Diabetes and Pregnancy Study Group (IADPSG) recommendations, which are widely applied in the hospitals of China. Briefly, all pregnant women were screened for GDM using the one-step 2-h 75-g OGTT. Women were diagnosed as having GDM once glucose values met or exceeded the diagnostic criteria: fasting glucose value $\geq 5.1 \mathrm{mmol} / \mathrm{L}, 1$-h glucose value $\geq 10.0 \mathrm{mmol} / \mathrm{L}$, and 2 - $\mathrm{h}$ glucose value $\geq 8.5 \mathrm{mmol} / \mathrm{L}$.

\section{Investigation content}

The following information was collected from medical records for each patient: maternal age, height, prepregnancy weight and body mass index (BMI), gestational weight gain, educational attainment $(\leq 12,13-16$, or $\geq 17$ years of completed schooling), family history of diabetes in the first-degree relatives, insulin treatment, mode of conception, mode of delivery, infant sex and weight, weight change between pregnancies, interpregnancy intervals, as well as biochemical parameters including FPG, uric acid, lipid profiles, glycated hemoglobin (HbA1c) and glucose values at the 75-g OGTT. All metabolic variables were measured at the first-trimester of pregnancy (12.0-14.0 gestational weeks) with the exception of the OGTT test and $\mathrm{HbA} 1 \mathrm{c}$ in the first pregnancy, which were measured at a mean of 26.5 gestational weeks. BMI was calculated as weight in kilograms divided by height in meters squared. Weight change between pregnancies was referred to the change in prepregnancy weight between the first and subsequent pregnancies. The interpregnancy intervals, defined as the number of months between the date of delivery of the first pregnancy and the date of last menstrual period of the subsequent pregnancy, was also calculated.

\section{Statistical analysis}

The data analyses were performed using SPSS 23.0 software (IBM SPSS, Chicago, IL, USA), and all variables were first assessed by normal distribution test (one-sample Kolmogorov-Smirnov test). If the data were normally distributed, we used an independent samples $t$-test for the continuous variables analysis. Dichotomous variables were evaluated with Chi-square test or Fisher's exact test (where $20 \%$ or more of the cells in a $x 2$ table will have an expected count less than 5). Univariate logistic regression analysis was used to compute each odds ratio (OR) and 95\% confidence interval (CI) and multivariate logistic regression analysis was used to identify independent predictors of GDM recurrence. Data are presented as the mean \pm standard error (SE) or number (percentage) for normally distributed data. A two-sided $p$ value less than 0.05 was considered statistically significant.

\section{Results}

\section{Frequency}

We collected the records of 142 primiparous women diagnosed with GDM in the first pregnancy; a total of 78 (55\%) women had recurrent GDM in subsequent pregnancy.

\section{Maternal characteristics in the first pregnancy}

Maternal characteristics in the first pregnancy are shown in Table 1. Demographic characteristics such as maternal age, prepregnancy weight and BMI, gestational weight 
Table 1 Maternal characteristics in the first pregnancy $(N=142)$

\begin{tabular}{|c|c|c|c|c|}
\hline Variables & $\begin{array}{l}\text { Recurrent GDM } \\
N=78\end{array}$ & $\begin{array}{l}\text { Primary GDM } \\
N=64\end{array}$ & $\begin{array}{l}\text { Unadjusted OR } \\
(95 \% \mathrm{Cl})\end{array}$ & $p$ value \\
\hline Age (years) & $30.0 \pm 0.3$ & $29.7 \pm 0.4$ & $1.05(0.93-1.17)$ & 0.442 \\
\hline Prepregnancy weight $(\mathrm{kg})^{\mathrm{a}}$ & $58.2 \pm 1.2$ & $58.8 \pm 1.3$ & $0.99(0.95-1.04)$ & 0.736 \\
\hline Prepregnancy BMI $\left(\mathrm{kg} / \mathrm{m}^{2}\right)^{\text {a }}$ & $22.8 \pm 0.5$ & $22.2 \pm 0.4$ & $1.07(0.94-1.22)$ & 0.320 \\
\hline Gestational weight gain $(\mathrm{kg})^{\mathrm{b}}$ & $11.7 \pm 0.8$ & $12.4 \pm 0.6$ & $0.97(0.88-1.07)$ & 0.510 \\
\hline \multicolumn{5}{|l|}{ Educational attainment (years) } \\
\hline$\leq 12$ & $7(9.0)$ & $6(9.4)$ & $1.02(0.32-3.23)$ & 0.790 \\
\hline $13-16$ & $54(69.2)$ & $47(73.4)$ & Ref & \\
\hline$\geq 17$ & $17(21.8)$ & $11(17.2)$ & $1.35(0.57-3.16)$ & \\
\hline Family history of first-degree diabetes & $20(25.6)$ & $11(17.2)$ & $1.66(0.73-3.79)$ & 0.228 \\
\hline Insulin treatment & $4(5.1)$ & $1(1.6)$ & $3.41(0.37-31.26)$ & 0.279 \\
\hline Conception by ART & $5(6.4)$ & $2(3.1)$ & $2.12(0.40-11.33)$ & 0.378 \\
\hline Delivery by Cesarean Section & $32(41.0)$ & $23(35.9)$ & $1.24(0.63-2.45)$ & 0.536 \\
\hline Birth weight (g) & $3264 \pm 55$ & $3300 \pm 47$ & $1.00(0.99-1.01)$ & 0.624 \\
\hline Male baby & $34(43.6)$ & $27(42.2)$ & $1.06(0.54-2.07)$ & 0.867 \\
\hline Biochemical parameters in first-trimester (weeks) & $13.3 \pm 0.2$ & $13.3 \pm 0.3$ & & \\
\hline Fasting plasma glucose (mmol/L) & $4.48 \pm 0.05$ & $4.66 \pm 0.05$ & $0.38(0.17-0.88)$ & $0.024^{*}$ \\
\hline Uric acid (umol/L) & $226 \pm 5$ & $214 \pm 5$ & $1.01(0.99-1.02)$ & 0.108 \\
\hline Cholesterol (mmol/L) & $5.01 \pm 0.09$ & $4.86 \pm 0.11$ & $1.28(0.84-1.95)$ & 0.250 \\
\hline Triglyceride (mmol/L) & $1.68 \pm 0.09$ & $1.40 \pm 0.07$ & $1.94(1.06-3.54)$ & $0.031^{*}$ \\
\hline 75-g OGTT in second-trimester (weeks) & $26.4 \pm 0.3$ & $26.6 \pm 0.4$ & & \\
\hline Fasting (mmol/L) & $4.62 \pm 0.07$ & $4.51 \pm 0.07$ & $1.40(0.77-2.52)$ & 0.269 \\
\hline $1 \mathrm{~h}(\mathrm{mmol} / \mathrm{L})$ & $10.44 \pm 0.17$ & $9.82 \pm 0.18$ & $1.34(1.05-1.71)$ & $0.018^{*}$ \\
\hline $2 \mathrm{~h}(\mathrm{mmol} / \mathrm{L})$ & $8.67 \pm 0.17$ & $8.55 \pm 0.20$ & $1.05(0.84-1.31)$ & 0.653 \\
\hline $\operatorname{HbA1c}(\%)^{c}$ & $5.4 \pm 0.1$ & $5.3 \pm 0.1$ & $3.00(1.03-8.78)$ & $0.045^{*}$ \\
\hline$[\mathrm{HbA} 1 \mathrm{c}(\mathrm{mmol} / \mathrm{mol})]$ & $36 \pm 1$ & $34 \pm 1$ & $1.11(1.00-1.22)$ & $0.045^{*}$ \\
\hline
\end{tabular}

Variables are presented with mean \pm standard error (SE) unless number (percentage)

GDM gestational diabetes mellitus, $O R$ odds ratio, $C l$ confidence interval, $B M I$ body mass index, $A R T$ assisted reproductive technology, OGTT oral glucose tolerance test, HbA1c glycated hemoglobin

a Prepregnancy weight and BMI: $32.4 \%$ missing;

${ }^{\mathrm{b}}$ Gestational weight gain: $41.5 \%$ missing;

c HbA1c: $26.1 \%$ missing

gain, educational attainment, mode of conception and delivery, birth weight and the infant's sex were comparable between the two groups. Although numbers were too small to detect potential significant differences, the proportions of family history of diabetes in first-degree relatives and insulin treatment were greater in the the group with GDM recurrence than the group with no recurrence. Biochemical parameters such as uric acid and cholesterol in the first-trimester, as well as fasting and postprandial 2-h glucose value at the OGTT test were also similar between the two groups. However, first-trimester FPG of the group with GDM recurrence was apparently lower, whereas triglyceride concentrations, postprandial 1-h glucose value and second-trimester $\mathrm{HbA1c}$ were significantly higher than the group with no recurrence.

\section{Maternal characteristics in the subsequent pregnancy} Maternal characteristics in the subsequent pregnancy are shown in Table 2. Demographic characteristics including maternal age, weight change between pregnancies and interpregnancy intervals showed no difference between the two groups, while the group with GDM recurrence manifested significantly higher prepregnancy BMI compared with the group with no recurrence. For biochemical parameters, there were not significant differences in first-trimester FPG, cholesterol, high-density lipoprotein and low-density lipoprotein cholesterol between the two groups. However, first-trimester HbA1c and triglyceride concentrations were significantly elevated in women who developed GDM in their subsequent pregnancy compared with women who did not. 
Table 2 Maternal characteristics in the subsequent pregnancy $(N=142)$

\begin{tabular}{|c|c|c|c|c|}
\hline Variables & $\begin{array}{l}\text { Recurrent GDM } \\
N=78\end{array}$ & $\begin{array}{l}\text { Primary GDM } \\
N=64\end{array}$ & $\begin{array}{l}\text { Unadjusted OR } \\
(95 \% \mathrm{Cl})\end{array}$ & $p$ value \\
\hline Age (years) & $32.6 \pm 0.3$ & $31.9 \pm 0.4$ & $1.09(0.97-1.21)$ & 0.144 \\
\hline Prepregnancy weight (kg) & $59.0 \pm 1.2$ & $57.2 \pm 1.0$ & $1.02(0.99-1.06)$ & 0.259 \\
\hline Prepregnancy BMI $\left(\mathrm{kg} / \mathrm{m}^{2}\right)$ & $22.9 \pm 0.4$ & $21.7 \pm 0.3$ & $1.12(1.00-1.25)$ & $0.043^{*}$ \\
\hline Weight change between pregnancies $(\mathrm{kg})^{\text {a }}$ & $0.7 \pm 0.7$ & $-0.3 \pm 0.7$ & $1.04(0.96-1.14)$ & 0.340 \\
\hline Interpregnancy intervals (months) & $22.6 \pm 1.1$ & $19.9 \pm 1.3$ & $1.03(0.99-1.06)$ & 0.135 \\
\hline Biochemical parameters in first-trimester (weeks) & $12.7 \pm 0.2$ & $12.6 \pm 0.2$ & & \\
\hline Fasting plasma glucose (mmol/L) & $4.76 \pm 0.07$ & $4.61 \pm 0.05$ & $1.87(0.93-3.74)$ & 0.078 \\
\hline $\mathrm{HbA} 1 \mathrm{c}(\%)^{\mathrm{b}}$ & $5.2 \pm 0.0$ & $5.1 \pm 0.0$ & $5.06(1.45-17.64)$ & $0.011^{*}$ \\
\hline $\mathrm{HbA1c}(\mathrm{mmol} / \mathrm{mol})$ & $34 \pm 0$ & $32 \pm 0$ & $1.16(1.04-1.30)$ & $0.011^{*}$ \\
\hline Cholesterol (mmol/L) & $5.11 \pm 0.11$ & $5.00 \pm 0.10$ & $1.14(0.79-1.64)$ & 0.486 \\
\hline Triglyceride (mmol/L) & $1.92 \pm 0.10$ & $1.53 \pm 0.07$ & $2.04(1.23-3.36)$ & $0.005^{*}$ \\
\hline $\mathrm{HDL}-\mathrm{C}(\mathrm{mmol} / \mathrm{L})$ & $1.57 \pm 0.04$ & $1.70 \pm 0.05$ & $0.38(0.14-1.01)$ & 0.052 \\
\hline LDL-C (mmol/L) & $3.28 \pm 0.10$ & $3.09 \pm 0.10$ & $1.35(0.88-2.09)$ & 0.172 \\
\hline
\end{tabular}

Variables are presented with mean \pm standard error (SE) unless number (percentage)

GDM gestational diabetes mellitus, $O R$ odds ratio, $C$ confidence interval, $B M I$ body mass index, $H b A 1 C$ glycated hemoglobin, $H D L-C$ high-density lipoprotein

cholesterol, LDL-C low-density lipoprotein cholesterol

a Weight change between pregnancies: $32.4 \%$ missing

b HbA1c: $4.2 \%$ missing

\section{Independent risk factors}

Potential risk factors were selected for inclusion in the multivariate logistic regression analysis to further examine the association with GDM recurrence. We included variables that were clinically important and/or statistically significant ( $p$ less than 0.05 ) between the two groups in the univariate logistic model. As shown in Table 3, the ORs were adjusted for maternal age and prepregnancy BMI in the first pregnancy, educational attainment, family history of diabetes, and insulin treatment. Notably, the first-trimester FPG and postprandial 1-h glucose value in the first pregnancy and first-trimester triglyceride concentrations in subsequent pregnancy were independent predictors of GDM recurrence.

\section{Discussion}

This study demonstrated that the frequency of recurrent GDM in subsequent pregnancies was $55 \%$ of all women who developed GDM in their first pregnancy at IPMCH during the study period, which was in accordance with the pooled GDM recurrence among Asian women according to a recent meta-analysis [11]. However, the frequency from our study, which mainly involved Han Chinese, was higher than the frequency of $45 \%$ reported by Kwak et al. [12], whose population was predominantly Korean. One of the explanations for this different recurrence rate is that the underlying incidence of GDM in the Chinese population [2] is greater than the Korean population [16] and the higher incidence of GDM possibly indicates elevated susceptibility to its recurrence. Another explanation might rely on the different diagnostic criteria, since IADPSG criteria may double the number of GDM diagnosed women [18] and give rise to a sum of mild GDM patients [19]. In contrast, the frequency from our study was lower than the reports of Nohira et al. [13], whose frequency was 66\%. Apart from the different diagnostic criteria, the Japanese

Table 3 Selected characteristics in association with the risk for GDM recurrence $(N=142)$

\begin{tabular}{lll}
\hline Variables & $\begin{array}{l}\text { Adjusted OR }{ }^{\text {a }} \\
(95 \% \mathrm{Cl})\end{array}$ & $p$ value \\
\hline First-trimester fasting plasma glucose in the first pregnancy & $0.24(0.10-0.63)$ & $0.004^{*}$ \\
First-trimester triglyceride in the first pregnancy & $1.77(0.96-3.27)$ & 0.069 \\
1 h glucose in the first pregnancy (75-g OGTT) & $1.43(1.09-1.87)$ & $0.010^{*}$ \\
First-trimester HbA1c in subsequent pregnancy & $3.59(0.93-13.88)$ & 0.063 \\
First-trimester triglyceride in subsequent pregnancy & $1.89(1.13-3.16)$ & $0.016^{*}$ \\
First-trimester HDL-C in subsequent pregnancy & $0.52(0.19-1.44)$ & 0.208 \\
\hline
\end{tabular}

${ }^{a}$ Adjusted for maternal age and prepregnancy BMI in subsequent pregnancy, educational attainment, family history of first-degree diabetes, and insulin treatment $O R$ odds ratio, $\mathrm{Cl}$ confidence interval, OGTT oral glucose tolerance test, HbA1c glycated hemoglobin 
study included both primiparous and multiparous women. Since primiparous women experienced a lower rate of GDM recurrence than multiparous women, the frequency will increased along with parity [11, 14, 19]. In addition, previous studies reported that the GDM frequencies ranged from 32 to $47 \%$ mainly based on Caucasian primiparous women $[11,20]$, which apparently lower than our results among Chinese primiparous women. This difference could be explained by ethnicity, as the frequency of GDM recurrence in Asians is higher than Caucasians [10, $11,20]$. Getahun et al. [21] suggested that large consumption of high glycemic index food and excess visceral adipose tissue accumulation might cause elevated levels of serum glucose and increase the risk of GDM among Asian populations. However, the women in our study had a mean prepregnancy BMI of 22.5 yet were susceptible to GDM despite not being overweight or obese. This fact highlights the difficulty in reducing the prevalence of GDM as more women developed western lifestyles associated with increasing wealth in China and globally.

Our findings were in agreement with previous studies $[14,19]$ which observed a positive association of high postprandial $1-\mathrm{h}$ glucose value in the first pregnancy with GDM recurrence. Additionally, postprandial 1-h glucose can serve as a better predictor for development of type 2 diabetes mellitus (T2DM) compared with 2-h glucose $[22,23]$. A probable reason is that the ability to utilize excess glucose in recurrent GDM patients decreases more severely than in primary GDM patients, which is similar to a study [23] that found that higher 1 -h glucose indicated the existence of severe IR. Furthermore, since the OGTT directly reflects the degree of glucose tolerance and indicates the risk of developing T2DM [24], there is no doubt that the higher levels of OGTT results predict the greater likelihood of progression to GDM recurrence. This speculation is supported by higher HbA1c concentration in the second trimester, because the degree of IR is significantly related to the increase of HbA1c [25]. Thus, it would be acceptable to determine that the postprandial 1-h glucose of the OGTT in the first pregnancy is associated with GDM recurrence. Interestingly, first-trimester FPG in the first pregnancy was remarkably lower in the group with GDM recurrence than that in the group with no recurrence and the correlation with recurrence was stronger than postprandial $1-\mathrm{h}$ glucose. It is widely accepted that a physiological reduction in FPG concentration occurs in normal pregnancy [26] and Zhu et al. [27] reported the median FPG among the Chinese population was $4.62 \mathrm{mmol} / \mathrm{L}$ at $12-14$ gestational weeks. In our study, the median FPG was characterized by a lower level (4.50 $\mathrm{mmol} / \mathrm{L}$ ) in the group with GDM recurrence, whereas the level for the group with no recurrence $(4.60 \mathrm{mmol} /$ L) was comparable to the average. However, women in both groups had similar prepregnancy BMI with lower FPG in the group with GDM recurrence in the first pregnancy. Whereas, the women in the GDM recurrence group now have a higher prepregnancy BMI and the FPG slightly higher than the group with no recurrence in the second pregnancy. Moreover, the jump in TG levels between pregnancies is higher in the group with GDM recurrence than in the group with no recurrence. Although weight change between pregnancies is not different statistically because of significant missing data, it is conceivable from the above observations that women in the group with GDM recurrence gained more weight and adipose tissue than the other group making them prone to GDM despite their lower FPG in the first pregnancy. As a consequence, we suggest that the relation to FPG and GDM is likely to be a statistical relationship rather than a real mechanistic one.

One of the key features in our study was that we examined whether GDM recurrence was related to lipid profiles. It is of interest to mention that the first-trimester triglyceride concentration in subsequent pregnancy was significantly higher in the group with GDM recurrence, which agrees with the predictive value of the GDM occurrence [28] and progression to diabetes after GDM [29]. The triglyceride level was positively associated with the area under the postpartum glucose curve in women with prior GDM according to clinical research studies [30, 31]. These findings indicate that a common pathophysiological foundation underlies these diseases, and normalization of triglyceride level should be included as a preventive therapy. In addition, patients from both groups manifested elevated triglycerides in subsequent pregnancy, but the relative growth rates were 14.3 and $9.3 \%$, for the GDM recurrence group and group with no recurrence, respectively. When circulating TG was at high levels, the formation of lipid droplets in the islet cells and the lipolysis of TG [32] were increased. On the one hand, the accumulation of TG causes cell toxicity and the number of islet cells decreases [33], which can aggravate IR and affect $\beta$-cell function. On the other hand, the risk of tissue exposure to free fatty acids (FFAs) increases, and high FFAs could result in IR via oxidative stress pathways [34]. That is, a higher triglyceride baseline in first pregnancy accompanied a greater increase in subsequent pregnancy, leading to double impairment for the group with GDM recurrence. Apart from the elevated serum triglycerides, a higher LDL-C (low-density lipoprotein cholesterol) level was observed in the group with GDM recurrence. A possible reason might correlate to the different nutrition structure and the amount of physical activity before subsequent pregnancy. Moses et al. [35] found that women with GDM recurrence had higher fat intake compared to those without recurrence according to their diet history and food records, which is consistent with our findings that 
higher LDL-C at the start of the subsequent pregnancy was a predictive factor for GDM recurrence. In addition, the group with GDM recurrence manifested lower HDL-C (high-density lipoprotein cholesterol) level than the group with no recurrence, which was also the strongest predictive factor of IR [36]. Therefore, early lipid profile derangement seems to have a critical impact on future glucose metabolism.

It is worth noting that we investigated risk factors for GDM recurrence among primiparous women in China. In addition, GDM recurrence patients were more likely to present higher prepregnancy BMI and lipid profiles such as LDL-C, which indicated that lifestyle intervention as soon as possible could reduce its recurrence.

Nevertheless, this study is not without limitations. First, the proportion of missing data was great including up to $32 \%$ of prepregnancy weights and $41 \%$ of gestational weight gains in first pregnancy, and approximately $32 \%$ of weight gains between pregnancies. Missing data were inevitable because the self-reporting of body weight was less accurate than measurement and records at the other hospitals were not always available, which may lead the weight change during consecutive pregnancies to become statistically insignificant between the two groups. Second, the missing data on the postpartum glucose tolerance test might have resulted in misdiagnosis of women with type 2 diabetes before the subsequent pregnancy and overestimated the GDM recurrence rate, which is also a common drawback in other studies $[10,11]$ due to general low compliance to conduct the postpartum diabetes screening. However, we strictly excluded patients suspected to have preexisting diabetes according to their medical records.

\section{Conclusions}

In summary, GDM recurred in more than half of the women in their subsequent pregnancy among primiparous women in China. The association of postprandial 1-h glucose values in the OGTT with the recurrence of GDM was confirmed based on our study. In addition, decreased first-trimester FPG in the first pregnancy and the dyslipidemia profile identified in this sample of Chinese women appeared to be novel risk factors for the development of subsequent GDM. Together, early examination of metabolic risk factors could aid in assessing women who are at high risk for GDM recurrence.

\section{Abbreviations}

BMI: Body mass index; Cl: Confidence interval; FPG: Fasting plasma glucose; GDM: Gestational diabetes mellitus; HbA1c: Glycated haemoglobin; HDLC: High-density lipoprotein cholesterol; IADPSG: International Association of the Diabetes and Pregnancy Study Group; IR: Insulin resistance; LDL-C: Lowdensity lipoprotein cholesterol; OGTT: Oral glucose tolerance test; OR: Odds ratio; T2DM: Type 2 diabetes mellitus

Acknowledgements

Not applicable.

\section{Funding}

This work was supported by the China-Canada Cooperation of the National Natural Science Foundation of China (81661128010); and Doctoral Innovation Fund of School of Medicine, Shanghai Jiao Tong University (BXJ201640).

\section{Availability of data and materials}

The datasets used and/or analysed during the current study are available from the corresponding author on reasonable request.

\section{Authors' contributions}

The first author WYY made contributions to analysis and interpretation of data, and been involved in drafting the manuscript. LY, LC and LJ helped me get the acquisition of data, sort out data, make sure the patients meet the grouping criteria and revised the original paper on grammatical errors. LXM, SJZ and HHF, as my supervisors and mentor, helped me design this casecontrol study and revised the paper critically on its structure, the main findings in Results section and how to better interpret the findings in Discussion section. Moreover, all the listed authors approved the revised version to be published and agreed to be accountable for all aspects of the work.

\section{Ethics approval and consent to participate}

This case-control study was approved by Ethics Committee of the International Peace Maternity and Child Health Hospital (Number: GKLW 2016-22).

The access to medical records should be approved by Director, Science and Education department and Medical Records department in sequential order. Each patient's personal information and medical record was also strictly protected.

The informed consents to all participants in our study were written in paper, and all of them gave their signatures and agreed to take part in this study.

Consent for publication

Not applicable.

\section{Competing interests}

The authors declare that they have no competing interests.

\section{Publisher's Note}

Springer Nature remains neutral with regard to jurisdictional claims in published maps and institutional affiliations.

\section{Author details}

${ }^{1}$ The International Peace Maternity and Child Health Hospital, Shanghai Jiao Tong University School of Medicine, No. 1961, Huashan Road, Shanghai 200030, China. ${ }^{2}$ The Key Laboratory of Reproductive Genetics (Ministry of Education), Women's Hospital School of Medicine Zhejiang University, Hangzhou 310058, People's Republic of China. ${ }^{3}$ Department of

Pathophysiology, Zhejiang University School of Medicine, Hangzhou 310058 , People's Republic of China.

Received: 10 April 2018 Accepted: 4 February 2019

Published online: 15 February 2019

\section{References}

1. Buchanan TA, Xiang AH. Gestational diabetes mellitus. J Clin Invest. 2005; 115(3):485-91

2. Yang H, Wei Y, Gao X, Xu X, Fan L, He J, Hu Y, Liu X, Chen X, Yang Z, et al. Risk factors for gestational diabetes mellitus in Chinese women: a prospective study of 16,286 pregnant women in China. Diabet Med. 2009; 26(11):1099-104

3. Zhu W, Yang H, Wei Y, Wang Z, Li X, Wu H, Li N, Zhang M, Liu X, Zhang H, et al. Comparing the diagnostic criteria for gestational diabetes mellitus of World Health Organization 2013 with 1999 in Chinese population. Chin Med J. 2015;128(1):125-7.

4. Lawrence JM, Contreras R, Chen W, Sacks DA. Trends in the prevalence of preexisting diabetes and gestational diabetes mellitus among a racially/ ethnically diverse population of pregnant women, 1999-2005. Diabetes Care. 2008:31(5):899-904.

5. Getahun D, Nath C, Ananth CV, Chavez MR, Smulian JC. Gestational diabetes in the United States: temporal trends 1989 through 2004. Am J Obstet Gynecol. 2008;198(5) 525 e521-525. 
6. Jovanovic L, Pettitt DJ. Gestational diabetes mellitus. JAMA. 2001;286(20): 2516-8.

7. Khambalia AZ, Ford JB, Nassar N, Shand AW, McElduff A, Roberts CL. Occurrence and recurrence of diabetes in pregnancy. Diabet Med. 2013; 30(4):452-6.

8. Kim C, Newton KM, Knopp RH. Gestational diabetes and the incidence of type 2 diabetes: a systematic review. Diabetes Care. 2002;25(10):1862-8.

9. Silverman $\mathrm{BL}$, Metzger $\mathrm{BE}, \mathrm{Cho} \mathrm{NH}$, Loeb CA. Impaired glucose tolerance in adolescent offspring of diabetic mothers. Relationship to fetal hyperinsulinism. Diabetes Care. 1995;18(5):611-7.

10. Kim C, Berger DK, Chamany S. Recurrence of gestational diabetes mellitus: a systematic review. Diabetes Care. 2007;30(5):1314-9.

11. Schwartz N, Nachum Z, Green MS. The prevalence of gestational diabetes mellitus recurrence--effect of ethnicity and parity: a metaanalysis. Am J Obstet Gynecol. 2015;213(3):310-7.

12. Kwak SH, Kim HS, Choi SH, Lim S, Cho YM, Park KS, Jang HC, Kim MY, Cho $\mathrm{NH}$, Metzger BE. Subsequent pregnancy after gestational diabetes mellitus: frequency and risk factors for recurrence in Korean women. Diabetes Care. 2008;31(9):1867-71.

13. Nohira T, Kim S, Nakai H, Okabe K, Nohira T, Yoneyama K. Recurrence of gestational diabetes mellitus: rates and risk factors from initial GDM and one abnormal GTT value. Diabetes Res Clin Pract. 2006;71(1):75-81.

14. Schwartz N, Nachum Z, Green MS. Risk factors of gestational diabetes mellitus recurrence: a meta-analysis. Endocrine. 2016;53(3):662-71.

15. Rao AK, Cheng YW, Caughey AB. Perinatal complications among different Asian-American subgroups. Am J Obstet Gynecol. 2006;194(5):e39-41.

16. Wong LF, Caughey AB, Nakagawa S, Kaimal AJ, Tran SH, Cheng YW. Perinatal outcomes among different Asian-American subgroups. Am J Obstet Gynecol. 2008;199(4):382 e381-6.

17. Bottalico JN. Recurrent gestational diabetes: risk factors, diagnosis, management, and implications. Semin Perinatol. 2007:31(3):176-84.

18. Ryan EA. Diagnosing gestational diabetes. Diabetologia. 2011;54(3):480-6.

19. Schwartz N, Green MS, Yefet E, Nachum Z. Modifiable risk factors for gestational diabetes recurrence. Endocrine. 2016;54(3):714-22.

20. Kruse AR, Darling MS, Hansen MK, Markman MJ, Lauszus FF, Wielandt HB. Recurrence of gestational diabetes in primiparous women. Acta Obstet Gynecol Scand. 2015;94(12):1367-72.

21. Getahun D, Fassett MJ, Jacobsen SJ. Gestational diabetes: risk of recurrence in subsequent pregnancies. Am J Obstet Gynecol. 2010;203(5):467 e461-6.

22. Leguizamon G, Krupitzki H, Glujovsky D, Olivera Ravasi M, Reece EA. Blood glucose monitoring in gestational diabetes mellitus: 1- versus 2-h blood glucose determinations. J Matern Fetal Neonatal Med. 2002;12(6):384-8.

23. Bianchi C, Miccoli R, Trombetta M, Giorgino F, Frontoni S, Faloia E, Marchesini G, Dolci MA, Cavalot F, Cavallo G, et al. Elevated 1-hour postload plasma glucose levels identify subjects with normal glucose tolerance but impaired beta-cell function, insulin resistance, and worse cardiovascular risk profile: the GENFIEV study. J Clin Endocrinol Metab. 2013;98(5):2100-5.

24. Solomon TP, Malin SK, Karstoft K, Knudsen SH, Haus JM, Laye MJ, Pedersen M, Pedersen BK, Kirwan JP. Determining pancreatic beta-cell compensation for changing insulin sensitivity using an oral glucose tolerance test. Am J Physiol Endocrinol Metab. 2014;307(9):E822-9.

25. Pan J, Zhang F, Zhang L, Bao Y, Tao M, Jia W. Influence of insulin sensitivity and secretion on glycated albumin and hemoglobin A1c in pregnant women with gestational diabetes mellitus. Int J Gynaecol Obstet. 2013;121(3):252-6.

26. Mills JL, Jovanovic L, Knopp R, Aarons J, Conley M, Park E, Lee YJ, Holmes L, Simpson JL, Metzger B. Physiological reduction in fasting plasma glucose concentration in the first trimester of normal pregnancy: the diabetes in early pregnancy study. Metabolism. 1998;47(9):1140-4.

27. Zhu WW, Yang HX, Wei YM, Yan J, Wang ZL, Li XL, Wu HR, Li N, Zhang MH, Liu XH, et al. Evaluation of the value of fasting plasma glucose in the first prenatal visit to diagnose gestational diabetes mellitus in China. Diabetes Care. 2013;36(3):586-90.

28. Wang C, Zhu W, Wei $Y$, Su R, Feng H, Hadar E, Hod M, Yang $H$. The associations between early pregnancy lipid profiles and pregnancy outcomes. J Perinatol. 2016.

29. Azar M, Stoner JA, Dao HD, Stephens L, Goodman JR, Maynard J, Lyons TJ. Epidemiology of Dysglycemia in pregnant Oklahoma American Indian women. J Clin Endocrinol Metab. 2015;100(8):2996-3003.

30. Pallardo F, Herranz L, Garcia-Ingelmo T, Grande C, Martin-Vaquero P, Janez M, Gonzalez A. Early postpartum metabolic assessment in women with prior gestational diabetes. Diabetes Care. 1999;22(7):1053-8.
31. Sokup A, Goralczyk B, Goralczyk K, Rosc D. Triglycerides as an early pathophysiological marker of endothelial dysfunction in nondiabetic women with a previous history of gestational diabetes. Acta Obstet Gynecol Scand. 2012:91(2):182-8.

32. Zhou M, Zhu L, Cui X, Feng L, Zhao X, He S, Ping F, Li W, Li Y. The triglyceride to high-density lipoprotein cholesterol (TG/HDL-C) ratio as a predictor of insulin resistance but not of beta cell function in a Chinese population with different glucose tolerance status. Lipids Health Dis. 2016;15:104.

33. Liang $Z$, Wu $Y$, Zhu $X$, Fang $Q$, Chen D. Insulin resistance and lipid profile during an oral glucose tolerance test in women with and without gestational diabetes mellitus. J Obstet Gynaecol. 2016;36(3):337-9.

34. Lam TK, Carpentier A, Lewis GF, van de Werve G, Fantus IG, Giacca A. Mechanisms of the free fatty acid-induced increase in hepatic glucose production. Am J Physiol Endocrinol Metab. 2003:284(5):E863-73.

35. Moses RG, Shand JL, Tapsell LC. The recurrence of gestational diabetes: could dietary differences in fat intake be an explanation? Diabetes Care. 1997;20(11):1647-50

36. Zheng $\mathrm{S}, \mathrm{Xu} \mathrm{H}$, Zhou H, Ren X, Han T, Chen Y, Qiu H, Wu P, Zheng J, Wang $L$, et al. Associations of lipid profiles with insulin resistance and beta cell function in adults with normal glucose tolerance and different categories of impaired glucose regulation. PLoS One. 2017:12(2):e0172221.

\section{Ready to submit your research? Choose BMC and benefit from:}

- fast, convenient online submission

- thorough peer review by experienced researchers in your field

- rapid publication on acceptance

- support for research data, including large and complex data types

- gold Open Access which fosters wider collaboration and increased citations

- maximum visibility for your research: over $100 \mathrm{M}$ website views per year

At $\mathrm{BMC}$, research is always in progress.

Learn more biomedcentral.com/submissions 